\title{
Sources of Variability in Performance Times at the World Orienteering Championships
}

\author{
KIM HÉBERT-LOSIER ${ }^{1}$, SIMON PLATT ${ }^{1}$, and WILLIAM G. HOPKINS ${ }^{2}$ \\ ${ }^{1}$ Swedish Winter Sports Research Centre, Department of Health Sciences, Mid Sweden University, Östersund, SWEDEN; \\ ${ }^{2}$ College of Sport and Exercise Science, Victoria University, Melbourne, AUSTRALIA
}

\begin{abstract}
HÉBERT-LOSIER, K., S. PLATT, and W. G. HOPKINS. Sources of Variability in Performance Times at the World Orienteering Championships. Med. Sci. Sports Exerc., Vol. 47, No. 7, pp. 1523-1530, 2015. Purpose: An improvement equal to 0.3 of the typical variation in an elite athlete's race-to-race performance estimates the smallest worthwhile enhancement, which has not yet been determined for orienteers. Moreover, much of the research in high-performance orienteering has focused on physical and cognitive aspects, although course characteristics might influence race performance. Analysis of race data provides insights into environmental effects and other aspects of competitive performance. Our aim was to examine such factors in relation to World Orienteering Championships performances. Methods: We used mixed linear modelling to analyze finishing times from the three qualification rounds and final round of the sprint, middle-distance, and long-distance disciplines of World Orienteering Championships from 2006 to 2013. Models accounted for race length, distance climbed, number of controls, home advantage, venue identity, round (qualification final), athlete identity, and athlete age. Results: Within-athlete variability (coefficient of variation, mean \pm SD) was lower in the final $(4.9 \% \pm 1.4 \%)$ than in the qualification $(7.3 \% \pm 2.4 \%)$ rounds and provided estimates of smallest worthwhile enhancements of $1.0 \%-3.5 \%$. The home advantage was clear in most disciplines, with distance climbed particularly impacting sprint performances. Small to very large between-venue differences were apparent. Performance predictability expressed as intraclass correlation coefficients was extremely high within years and was high to very high between years. Age of peak performance ranged from 27 to $31 \mathrm{yr}$. Conclusions: Our results suggest that elite orienteers should focus on training and strategies that enhance performance by at least $1.0 \%-3.5 \%$ for smallest worthwhile enhancement. Moreover, as greater familiarity with the terrain likely mediated the home advantage, foreign athletes would benefit from training in nations hosting the World Orienteering Championships for familiarization. Key Words: ATHLETIC PERFORMANCE, FOOT ORIENTEER, LINEAR MODELS, STATISTICS, RELIABILITY
\end{abstract}

0 uccessful foot orienteering performance relies on both running and navigational skills. In this sport, the winner is the orienteer who runs to a sequenced number of controls the fastest, using a map and compass to navigate over an unmarked course to locate these controls. Among the several races organized each year, the World Orienteering Championships (WOC) are the most prestigious. To compete in the final round for the World Champion title in the sprint, middle-distance, or long-distance event, each athlete must first finish at the top of his/her qualification round.

In elite sport, an improvement equal to 0.3 of the typical variation in an athlete's race-to-race performance estimates the smallest worthwhile enhancement in performance and reflects winning one more medal per 10 competitions (24).

Address for correspondence: Kim Hébert-Losier, Ph.D., P.T., Swedish Winter Sports Research Centre, Department of Health Sciences, Mid Sweden University, Kunskapens väg 8, Hus D, 83125 Östersund, Sweden; E-mail: kim.hebert-losier@miun.se.

Submitted for publication July 2014.

Accepted for publication October 2014.

0195-9131/15/4707-1523/0

MEDICINE \& SCIENCE IN SPORTS \& EXERCISE E

Copyright $(C) 2014$ by the American College of Sports Medicine

DOI: $10.1249 / \mathrm{MSS} .0000000000000558$
This within-athlete variability is usually expressed as a coefficient of variation (CV) and has been determined for several individual sporting events, including cross-country skiing $(1.1 \%-1.8 \%)(41)$, cycling $(0.4$ to $2.9 \%)(37)$, track and field (1.3\%-2.2\%) (23), and long-distance running (1.5\%-3.8\%) (25). Although foot orienteering involves a large running component, it is unique in terms of physical and mental effort, thus cautioning against direct generalization from other runningbased sports. Establishing the within-athlete variability for orienteers is important to determine what constitutes the smallest worthwhile enhancement in this sport, which could help guide clinical decision making related to training strategies and interventions.

To date, much of the research on high-performance orienteering has focused on physical $(21,28,31)$ or cognitive $(14,34)$ factors. At the same time, each orienteering race is unique with respect to race length and terrain, with variable amounts of ascent, descent, and number of controls. Course characteristics, such as course profiles, can exert an effect on orienteering finishing times. Many of these factors are important aspects in course planning and taken into consideration by an orienteer when deciding what route to take (12). The course length, number of controls, and distance climbed are the key course characteristics documented at the WOC. Knowledge regarding the effect of course layout on finishing 
times could provide valuable information to course setters, permitting them to develop a course that closely meets the International Orienteering Federation's competition rules (18).

Moreover, under a balanced "home-and-away" schedule, the home teams in sport competitions win more than $50 \%$ of games played, a phenomenon referred to as the "home advantage" (11). Both psychological and physiological factors mediate this home advantage (6), which, although to a smaller extent (29), also benefits athletes competing in individual sports and nations hosting international competitions (7). Although no home advantage has yet been documented in the sport of orienteering, its existence would corroborate with the small, but noteworthy, improvements in performance times of high school cross-country runners competing at home (35). The presence of a clear home advantage might encourage national teams to incorporate several familiarization runs at foreign championships venues.

Investigating relations between age and peak performance is another area of interest in elite sport (16). Bird et al. (3) concluded little effect of age on orienteering speed from 21 to $40 \mathrm{yr}$ of age based on results from four British orienteering competitions. Despite informing on overall performance trends with age at a national level, this investigation provides little insight into age of peak performance at international events, such as the WOC. Information pertaining to the age of peak performance in each orienteering event could be useful in the long-term planning of a competitive orienteering career and guide decision making relating to specialization in the sprint, middle-distance, or long-distance events.

With these considerations in mind, the results from the 2006 to 2013 WOC were analyzed using a mixed linear modeling approach. Our aim was to assess the within-athlete variability; the between-athlete, between-venue, and between-round differences in performance; the effects of course characteristics on finishing times; the ages of peak performance; and the predictability of performance in the sprint, middle-distance, and long-distance WOC races. Our findings should be of considerable interest to-among others - orienteering athletes, coaches, race organizers, and sportscasters.

\section{METHODS}

Data extraction. At the WOC, there are individual sprint, middle-distance, and long-distance events and a three-person team relay for males and females. Before 2014, all competitors in individual events needed to compete in one of three qualification rounds, with the top 15 athletes of each round proceeding to a single final event. In this study, finishing times from the qualification and final rounds of the sprint, middle-distance, and long-distance individual events from 2006 to 2013 were considered, implicating 24 finals $(8 \mathrm{WOC} \times 3$ events $\times 1$ final round $\times 2$ sexes) and 72 qualifications ( 8 WOC $\times 3$ events $\times 3$ qualification rounds $\times 2$ sexes). Each WOC was held at a unique location, hosted sequentially by Denmark, Ukraine, Czech Republic, Hungary, Norway, France, Switzerland, and Finland from 2006 to 2013.
The official WOC results from 2006 to 2013 were obtained from the Web sites of each annual organizing committee, which are linked to the International Orienteering Federation's Web site (http://orienteering.org/). When results were no longer available through such means, the organizing committee was contacted directly. In addition to the official finishing times and ranks of each athlete; data on the length, amount of climb, and number of controls for each race course were extracted and kept for analyses to assess the effect of course characteristics, as were the nationality of athletes and location of WOC to evaluate the home advantage. The dates of birth of athletes were also extracted when available, and data were complemented by searching an online orienteering database (http://runners.worldofo.com/).

Data were compiled for analysis using Excel 2007 (Microsoft Corporation, Redmond, WA) and statistically analyzed using Statistical Analysis System (Version 9.4; SAS Institute, Inc., Cary, NC). Written informed consent from individuals was not sought because no individuals were named, and all data used in this project were in the public domain.

Statistical analysis. Descriptive summaries of the data are presented using mean and SD values. For inferential analyses, mixed linear modeling procedures similar to those used to assess cross-country skiing (41), rowing (40), and longdistance running (25) performances were developed and allowed only positive variances. We used linear mixed modelling (Proc Mixed in SAS) to estimate effects on performance time based on the restricted maximal likelihood method. Our linear models permitted estimation of the effects of environmental conditions (i.e., race course characteristics) on orienteering performance adjusted for differences and changes in athlete performance and permitted estimation of differences and changes in athlete performance adjusted for differences in environmental conditions. The mixed model with restricted maximum likelihood estimation permitted estimation of several sources of withinathlete variation (here within- and between-year random error) when athletes entered a limited number of competitions.

To establish the smallest worthwhile enhancement in performance (i.e., 0.3 of the within-athlete variability) and the effect of course characteristics, a mixed linear model was developed and applied to the finishing times of qualification rounds and final rounds separately. The fixed effects in this model were race length (numeric), distance climbed (numeric), number of controls (numeric), and home advantage (two levels: yes, no). The random effects were athlete identity (to estimate the pure between-athlete differences), venue (to estimate the between-venue or year-to-year differences), and the residual (to estimate the within-athlete year-to-year variability). For the analysis of qualification rounds, the random effects also included venue $\times$ round (to estimate the between-round differences at any given venue). Race finishing times, race length, distance climbed, and number of controls were log-transformed before analysis to limit bias arising from the non-uniformity of error (1) and to yield variability and differences in performance times as percentages of means after back-transformation (CV; percent effects, \%) (26). Separate analyses were performed for 
each sex (male and female) and event (sprint, middle, and long), with the uncertainty in all estimates expressed using $90 \%$ confidence limits $(\mathrm{CL})$ in a plus/minus $( \pm)$ or time/divide $(\times / \div)$ form (i.e., $\mathrm{CV}, \pm \mathrm{CL}$ or $\mathrm{CV}, \times / \div \mathrm{CL}$ ). A $90 \%$ level was chosen for CL because the chances that the true value is smaller than the lower limit or greater than the upper limit are both $5 \%$, which is interpreted as very unlikely and is regarded as adequate precision for nonclinical estimates of true values (26). Only data from athletes who participated in at least two WOC were analyzed because only they contributed to the estimate of the within-athlete variability between championships, that is, between years.

To investigate the age of peak performance, the mixed linear model was tailored to analyze the performance times from the qualification rounds and final rounds together, thereby increasing the level of precision. In this second model, round (two levels: qualification, final) was added as a fixed effect and athlete $\times$ venue was added as a random effect (to estimate the within-athlete year-to-year variability between venues), with the residual now reflecting the within-athlete qualificationto-final variability at a given venue within years. Fixed effects for age (numeric) and age-squared (numeric) were also added, defining the effect of age as a quadratic. Age of peak performance was estimated from the outputs by dividing the negative age-squared coefficient by twice that of the age coefficient. The $90 \%$ CL were estimated with the following novel semiparametric bootstrapping method: the SE for the quadratic $(a)$ and linear coefficients for age $(b)$ and for their covariance were each combined with unit normally distributed random numbers to generate 10,000 simulated samples of $a, b$, and peak age ( $-b / 2 a)$; the CL were then provided by the 5 th and 95 th percentiles of the sample values for peak age.

In the first run of the mixed linear models, five performance times had standardized residuals above 5.0 that represented unusually slow times. Because these slow times were likely reflecting athletes getting lost during a race, these outliers were removed and data were reanalyzed.

Thresholds for interpreting magnitudes of the effects of race course characteristics on mean performance times as being small, moderate, large, very large, and extremely large were respectively $0.3,0.9,1.6,2.5$, and 4.0 of the withinathlete final-to-final (residual) $\mathrm{CV}$ determined for each sex and discipline (and trivial when below 0.3). These thresholds represent enhancements that would provide a top athlete with one, three, five, seven, and nine extra medals per 10 races (26). CV representing typical differences in the overall mean finishing times between venues and within venues between rounds were doubled before interpreting their magnitudes (26). An effect was deemed "clear" when its CL did not overlap the thresholds for small positive and small negative effects. For clarity in the tables, we have introduced a novel method to highlight clear effects and their magnitudes using superscript letters: ${ }^{\mathrm{T}}$, trivial; ${ }^{\mathrm{S}}$, small; ${ }^{\mathrm{M}}$, moderate; ${ }^{\mathrm{L}}$, large; $\mathrm{v}$, very large; and ${ }^{\mathrm{X}}$, extremely large.

Predictability of performance was expressed as intraclass correlation coefficients (ICC), which are effectively test-retest correlation coefficients that define the relative reproducibility of measurements; they were derived from the first set of mixed linear models (i.e., without age). The within-year ICC was calculated in analyses combining the qualification and the final rounds as the pure between-athlete variance (sum of the variances represented by athlete and athlete $\times$ venue random effects) divided by the observed between-athlete variance (sum of the pure between-athlete variance and the within-athlete variance represented by the mean residual) at a given venue. This ICC represents the correlation for an athlete's times between a qualification and a final round. The between-year ICC was calculated in separate analyses for the qualification and final rounds as the pure between-athlete variance (represented by the athlete random effect alone) divided by the observed between-athlete variance (sum of the pure between-athlete variance and the within-athlete variance represented by the mean residual). These ICC represent the correlation for an athlete's times between any two given championships. Confidence limits for each ICC were derived assuming that the within- to between-athlete ratio of the sample-to-population variance ratio had an $F$ sampling distribution. The magnitude of the ICC was assessed using a set of thresholds that assumed a 2-SD difference in performance between athletes in one race that predicts differences in performance between those athletes in another race. The threshold values of the ICC were $0.14,0.36,0.54,0.69$, and 0.83 for low, moderate, high, very high, and extremely high, respectively (40).

\section{RESULTS}

From 2006 to 2013, a total of 2068 different athletes participated in at least one WOC event and a total of 6602 individual races. Of these athletes, 1041 competed in at least two championships, providing 5295 individual races for mixed modeling analyses. Each male discipline contained 923 to 1007 observations; and each female discipline, 782 to 849 . The typical athlete competed in $3.4 \pm 1.5 \mathrm{WOC}$ (mean \pm SD).

Sources of variability. The characteristics of the WOC qualification and final rounds are summarized in Table 1. Of note are systematically longer finishing times, race length, distance climbed, and number of controls in the final rounds than the qualification rounds for the long- and middle-distance events. As shown in Table 2, the within-athlete variability across disciplines was consistently lower in the final rounds compared to the qualification rounds $(4.9 \% \pm 1.4 \%$ vs $7.3 \% \pm$ $2.4 \%$, mean $\pm \mathrm{SD})$ and in males compared to females $(5.4 \% \pm$ $1.7 \%$ vs $6.9 \% \pm 2.7 \%$ ). The between-athlete differences showed similar trends, with smaller $\mathrm{CV}$ in the final rounds $(6.1 \% \pm 2.0 \%$ vs $13.6 \% \pm 3.7 \%)$ and male races $(9.3 \% \pm 5.9 \%$ vs $10.4 \% \pm 4.0 \%$ ). On the basis of the within-athlete variation, the smallest worthwhile enhancement in performance ranged from $0.96 \%$ to $2.1 \%(1.5 \% \pm 0.4 \%$, mean $\pm \mathrm{SD})$ for final rounds and from $1.6 \%$ to $3.5 \%(2.2 \% \pm 0.7 \%)$ for qualification rounds. On average, these values were $1.3 \%$ and $1.6 \%$ in the final rounds and $1.9 \%$ and $2.5 \%$ in the 
TABLE 1. Race characteristics (mean \pm SD) from the qualification heats $(n=24, \mathrm{Q})$ and final rounds $(n=8$, F) of the World Orienteering Championships from 2006 to 2013.

\begin{tabular}{|c|c|c|c|c|c|c|c|}
\hline & & \multicolumn{2}{|c|}{ Sprint } & \multicolumn{2}{|c|}{ Middle } & \multicolumn{2}{|c|}{ Long } \\
\hline & & Female & Male & Female & Male & Female & Male \\
\hline \multirow[t]{2}{*}{ Finishing time (min) } & Q & $16.4 \pm 2.3$ & $15.6 \pm 1.9$ & $37.0 \pm 2.9$ & $33.8 \pm 2.6$ & $60.3 \pm 4.2$ & $74.0 \pm 6.0$ \\
\hline & $\mathrm{F}$ & $16.1 \pm 1.6$ & $15.9 \pm 1.1$ & $42.1 \pm 2.9$ & $40.2 \pm 2.5$ & $95.2 \pm 5.7$ & $113.8 \pm 7.5$ \\
\hline \multirow[t]{2}{*}{ Race length (km) } & $Q$ & $2.8 \pm 0.4$ & $3.1 \pm 0.4$ & $3.8 \pm 0.5$ & $4.5 \pm 0.6$ & $6.9 \pm 1.0$ & $10.4 \pm 1.4$ \\
\hline & $\mathrm{F}$ & $2.8 \pm 0.5$ & $3.2 \pm 0.6$ & $4.9 \pm 0.5$ & $6.1 \pm 0.5$ & $11.7 \pm 1.2$ & $17.4 \pm 1.5$ \\
\hline \multirow[t]{2}{*}{ Distance climbed (m) } & $Q$ & $57 \pm 40$ & $65 \pm 44$ & $135 \pm 46$ & $161 \pm 44$ & $246 \pm 58$ & $388 \pm 69$ \\
\hline & $\mathrm{F}$ & $58 \pm 26$ & $73 \pm 34$ & $193 \pm 50$ & $239 \pm 58$ & $451 \pm 91$ & $681 \pm 138$ \\
\hline \multirow[t]{2}{*}{ No. controls } & $Q$ & $15.1 \pm 2.8$ & $17.2 \pm 3.5$ & $13.0 \pm 3.2$ & $14.3 \pm 2.7$ & $13.5 \pm 2.3$ & $20.1 \pm 2.7$ \\
\hline & $\mathrm{F}$ & $18.0 \pm 2.6$ & $19.9 \pm 3.1$ & $18.4 \pm 3.5$ & $21.5 \pm 3.3$ & $23.5 \pm 1.7$ & $31.5 \pm 2.7$ \\
\hline
\end{tabular}

qualification rounds for males and females. $\mathrm{CV}$ representing differences in finishing times were large to very large between venues for the final rounds $(6.2 \% \pm 1.2 \%$, range $=$ $4.5 \%-7.9 \%)$ and small to very large for the qualification rounds $(6.8 \% \pm 2.2 \%$, range $=3.1 \%-9.1 \%)$. Across disciplines, the differences in finishing times between qualification rounds within a venue was small $(1.8 \% \pm 0.7 \%)$.

Effect of course characteristics. Table 3 presents estimates of the effect of course characteristics on the finishing times of qualification and final rounds. Home advantage had a mean effect of $-3.3 \% \pm 1.8 \%$ on the performance times and was clearly beneficial in most disciplines.

Increasing the amount of climb by $50 \mathrm{~m}$ had a large to very large slowing effect on sprint times $(11.9 \% \pm 5.8 \%)$ but small to trivial effects on middle and long events' times $(1.2 \% \pm 2.1 \%)$. Adding five controls had inconsistent effects on the mean performance times $(-2.4 \% \pm 6.8 \%)$. Lengthening a course by $20 \%$ caused trivial to large changes in performance times $(4.9 \% \pm 5.9 \%)$, with clear changes in the middle finals for both males and females, as well as all but one qualification round.

Age of peak performance. Age was available for 916 of the 1041 athletes, providing age of athletes on race day for 4864 races. In a typical race, the age of athletes was $27 \pm 5 \mathrm{yr}$ (mean $\pm \mathrm{SD}$ ), with the youngest and oldest athletes being 17 and 48 yr. The age of peak performance determined from these data ranged from 26.6 to $30.9 \mathrm{yr}$, with $\pm 90 \%$ CL from 2.5 to 8.2 yr. The quadratic age trends and estimates for each event and sex are plotted in Figure 1. Overall, the age of peak performance was $2.2 \mathrm{yr}$ earlier in male than in female athletes and $2.1 \mathrm{yr}$ earlier in sprint than in middle-distance and long-distance races.

Predictability of performance. Between years, the mean predictability of performance was higher in the qualification rounds (very high predictability) than the final rounds (high predictability), with ICC of $0.77 \pm 0.07$ (mean \pm SD; range $=$ $0.68-0.87$ ) and $0.59 \pm 0.10$ (range $=0.46-0.72$ ), respectively. Predictability of performance within years was extremely high across disciplines, with ICCs ranging from 0.87 to 0.94 .

\section{DISCUSSION}

The mixed linear model analyses documented here provide novel insights into the variability and predictability of performance of athletes competing in the WOC, as well as estimates of the smallest worthwhile effects for WOC performance enhancement $(0.96 \%-3.5 \%)$. Moreover, there was a small to moderate home advantage in most WOC disciplines, as well as small to very large between-venue differences in finishing times. The extremely high within-venue predictability of orienteering performance indicates that the most successful athletes during qualification rounds are the most successful in subsequent final rounds. In contrast, the betweenyear predictability of performance was lower, indicating less consistency in finishing times and rankings of individuals between WOC, especially in the final rounds. Finally, to our knowledge, we provide the first estimates of the age of peak performance for the various orienteering disciplines (27-31 yr).

The within-athlete variability of performance in final rounds of WOC estimated here $(3.2 \%-7.0 \%)$ is greater than that reported for final rounds in cross-country skiing $(1.5 \%$ $1.8 \%$ ) (41), cycling $(0.4 \%-2.9 \%)$ (37), track and field $(1.3 \%-2.2 \%)(23)$, and cross-country running $(1.5 \%-3.8 \%)$ (25). The greater variability in orienteering is likely due to race outcomes depending on a combination of physical (running) and mental (navigational) abilities, wherein navigational errors can substantially hinder finishing times. Moreover, information pertaining to several course characteristics and

TABLE 2. Estimates of within-athlete variability and pure differences between athletes, between venues, and between qualification heats within a venue (expressed as coefficients of variation, \%) in finishing times from qualification heats $(n=24, Q)$ and final rounds $(n=8, \mathrm{~F})$ of the World Orienteering Championships from 2006 to 2013.

\begin{tabular}{|c|c|c|c|c|c|c|c|}
\hline & & \multicolumn{2}{|c|}{ Sprint } & \multicolumn{2}{|c|}{ Middle } & \multicolumn{2}{|c|}{ Long } \\
\hline & & Female & Male & Female & Male & Female & Male \\
\hline \multirow[t]{2}{*}{ Within athlete } & $Q$ & 5.5 & 5.2 & 11.5 & 8.0 & 8.1 & 5.6 \\
\hline & $\mathrm{F}$ & 4.1 & 3.2 & 7.0 & 6.0 & 5.2 & 4.1 \\
\hline \multirow[t]{2}{*}{ Between athletes } & $Q$ & 9.2 & 9.2 & 16.7 & 18.2 & 13.8 & 14.3 \\
\hline & $\mathrm{F}$ & 6.1 & 3.0 & 8.2 & 6.5 & 8.3 & 4.8 \\
\hline \multirow[t]{2}{*}{ Between venues } & $Q$ & $7.9^{\mathrm{V}}$ & $9.1^{\mathrm{V}}$ & $6.8^{\mathrm{M}}$ & $5.5^{\mathrm{M}}$ & 3.1 & $8.6^{\mathrm{V}}$ \\
\hline & $\mathrm{F}$ & $7.4^{\mathrm{V}}$ & $4.5^{\mathrm{V}}$ & $5.9^{\mathrm{L}}$ & $5.6^{\mathrm{L}}$ & $5.8^{\mathrm{L}}$ & $7.9^{\mathrm{V}}$ \\
\hline Between heats & $Q$ & $1.0^{\mathrm{S}}$ & $0.8^{\mathrm{s}}$ & $2.4^{\mathrm{S}}$ & $2.3^{\mathrm{S}}$ & $2.2^{\mathrm{S}}$ & $1.8^{\mathrm{S}}$ \\
\hline
\end{tabular}

Uncertainty $(90 \%$ limits, $\times / \div)$ : within athlete, 1.06-1.10; between athletes, 1.10-1.19; between venues, 1.73-3.40; between heats, 1.66-4.04.

Superscript letters denote the magnitude of clear between-venues and between-heats estimates: ${ }^{\mathrm{T}}$, trivial; ${ }^{\mathrm{S}}$, small; ${ }^{\mathrm{M}}$, moderate; ${ }^{\mathrm{L}}$, large; ${ }^{\mathrm{V}}$, very large; and ${ }^{\mathrm{X}}$, extremely large. 
TABLE 3. Percent effects (mean, $\pm 90 \%$ confidence limits) of course characteristics on performance times in the qualification heats $(n=24, Q)$ and final rounds ( $n=8$, F) of the World Orienteering Championships from 2006 to 2013.

\begin{tabular}{|c|c|c|c|c|c|c|c|}
\hline & & \multicolumn{2}{|c|}{ Sprint } & \multicolumn{2}{|c|}{ Middle } & \multicolumn{2}{|c|}{ Long } \\
\hline & & Female & Male & Female & Male & Female & Male \\
\hline \multirow[t]{2}{*}{$20 \%$ distance } & $Q$ & $8.5, \pm 5.6^{\mathrm{M}}$ & $5.0, \pm 6.3^{\mathrm{M}}$ & $7.5, \pm 11.1^{\mathrm{s}}$ & $12.8, \pm 7.4^{\mathrm{M}}$ & $7.5, \pm 4.7^{\mathrm{S}}$ & $-3.4, \pm 8.4$ \\
\hline & $\mathrm{F}$ & $3.1, \pm 7.0$ & $0.7, \pm 4.1$ & $11.7, \pm 11.7^{\mathrm{M}}$ & $10.3, \pm 12.4^{\mathrm{L}}$ & $1.0, \pm 9.4$ & $-5.6, \pm 17.6$ \\
\hline \multirow[t]{2}{*}{ 50-m climb } & $Q$ & $20.5, \pm 9.1^{v}$ & $10.3, \pm 6.2^{\mathrm{L}}$ & $-1.8, \pm 5.3$ & $0.3, \pm 4.4$ & $1.7, \pm 2.4^{\top}$ & $0.6, \pm 2.4$ \\
\hline & $\mathrm{F}$ & $8.3, \pm 12.4$ & $8.6, \pm 6.1^{\mathrm{V}}$ & $3.9, \pm 7.5$ & $4.5, \pm 5.1^{\mathrm{s}}$ & $0.3, \pm 3.0$ & $-0.3, \pm 3.0$ \\
\hline \multirow[t]{2}{*}{ Five controls } & $Q$ & $-1.5, \pm 5.3$ & $-0.5, \pm 4.5$ & $-6.8, \pm 11.9$ & $-11.7, \pm 8.3^{\mathrm{M}}$ & $-10.5, \pm 6.3^{\mathrm{M}}$ & $5.6, \pm 7.0^{\mathrm{M}}$ \\
\hline & $\mathrm{F}$ & $10.7, \pm 14.0$ & $-0.1, \pm 6.1$ & $-4.4, \pm 9.1$ & $-2.6, \pm 8.1$ & $-10.1, \pm 14.2$ & $3.1, \pm 15.3$ \\
\hline \multirow[t]{2}{*}{ Home advantage } & $Q$ & $0.5, \pm 2.9$ & $-2.0, \pm 2.1^{\mathrm{S}}$ & $-4.8, \pm 4.4^{\mathrm{S}}$ & $-5.2, \pm 3.7^{\mathrm{s}}$ & $-3.6, \pm 3.3^{\mathrm{S}}$ & $-3.2, \pm 2.4^{\mathrm{S}}$ \\
\hline & $\mathbf{F}$ & $-4.1, \pm 2.2^{\mathrm{M}}$ & $-3.3, \pm 1.4^{\mathrm{M}}$ & $-1.5, \pm 3.0^{\top}$ & $-5.5, \pm 2.8^{\mathrm{M}}$ & $-2.0, \pm 2.2^{\mathrm{S}}$ & $-5.2, \pm 1.9^{\mathrm{M}}$ \\
\hline
\end{tabular}

Superscript letters denote the magnitude of clear effects of course characteristics: ${ }^{\mathrm{T}}$, trivial; ${ }^{\mathrm{S}}$, small; ${ }^{\mathrm{M}}$, moderate; ${ }^{\mathrm{L}}$, large; ${ }^{\mathrm{V}}$, very large; and ${ }^{\mathrm{X}}$, extremely large.

environmental factors with the potential of affecting orienteering performance was not readily accessible or consistently documented between WOC venues. Therefore, our model could not adjust for factors pertaining to terrain composition, altitude, or temperature and their effect on the athletes' performances, which likely caused our analyses to overestimate the within-athlete variability, as such factors can influence elite sports performance $(22,23,44)$. Indeed, changes in environments between venues would favor certain athletes more than others based on their relative strengths, for example, athletes skilled at running in rough-forest terrain would perform better on a predominantly forested rather than asphalted course. Furthermore, our within-athlete variability reflects the typical year-to-year difference in performance rather than the more commonly reported within-year competition-tocompetition variability $(23,25,41)$. Our estimate also represents individual differences in performance between any two given WOC from 2006 to 2013 and not the pure difference between consecutive years of competition. In consequence, the smallest worthwhile enhancement from one year to the next would be slightly smaller than the one reported here.

In the present study, the top third orienteers (i.e., those participating in final rounds) demonstrated a superior yearto-year consistency and homogeneity in performance than the full group. More precisely, final rounds exhibited lower within-athlete variability and between-athlete differences in finishing times than corresponding qualification rounds, in agreement with observations from other sports $(25,41)$. The greater consistency observed in the higher-performing orienteers likely stems from their better conditioning (21), higher preparedness state (30), as well as an advanced ability to attend to the map while running (15). The smaller between-athlete differences in the finals reflect the narrower range in abilities of higher-performing orienteers. Similar to the within-athlete variability, not all factors with the potential to influence between-athlete differences were accounted for in our statistical model (e.g., athlete-related characteristics, including years of competition), leading to an inflation of estimates.

On the basis of the within-athlete variation, an enhancement in performance of $0.96 \%-2.1 \%$ in a top-level orienteer would be needed to win one more medal per 10 competitions, whereas an increment of $1.6 \%-3.5 \%$ would allow an orienteer to finish in the top of their qualification rounds more often and participate in the final rounds. Therefore, interventions or training programs that entail such enhancements in performance can be recommended for use to orienteering athletes and coaches. For instance, Kujala et al. (32) have identified that ingesting glucose polymer syrup in addition to a $2.5 \%$ glucose solution before and during a prolonged orienteering competition benefits performance by approximately $5.5 \%$, hence meeting the threshold of smallest worthwhile change. Similarly, a more recent investigation determined that elite orienteers who ingested a carbohydrate beverage containing whey protein hydrolysate before and after each training session during a 1-wk training camp improved their 4-km time-trial performances by approximately $2 \%$ by the end of the week compared to those orienteers who had ingested a carbohydrate-only beverage (20), also meeting the threshold for smallest worthwhile enhancement in performance.

The female athletes exhibited greater within-athlete variability and between-athlete differences in performance than their male counterparts. The sex difference observed here may be due to a lesser depth of competition in females than to physiological (9), including hormonal (33), variations. Indeed, there were fewer observations for mixed modelling analysis for female than male athletes, indicating that a smaller proportion of females competed at multiple championships. Furthermore, males generally outperform females in orientation tasks (10), which might contribute to the higher consistency in performance in male orienteers, as well as their earlier age of peak performance.

Clear small to moderate improvements in performance times were detected when racing at home in most disciplines. The factors contributing to the home advantage in orienteering are likely numerous and, along with crowd and travel factors (8), presumably include a greater familiarity with the terrain, vegetation, and climate. These findings also support that national teams preparing for WOC hosted in a foreign country would benefit from training in the destined location. That the home advantage was generally more pronounced in the middledistance and long-distance events rather than the sprint partly supports a role for terrain and vegetation in the home advantage. Because sprints are contested over runnable park or urban terrain (i.e., on asphalt) (18), conditions are generally reproducible between venues in contrast to the middle-distance and long-distance race courses that are ran off road on more technically complex terrain (18) and therefore less reproducible between venues. 

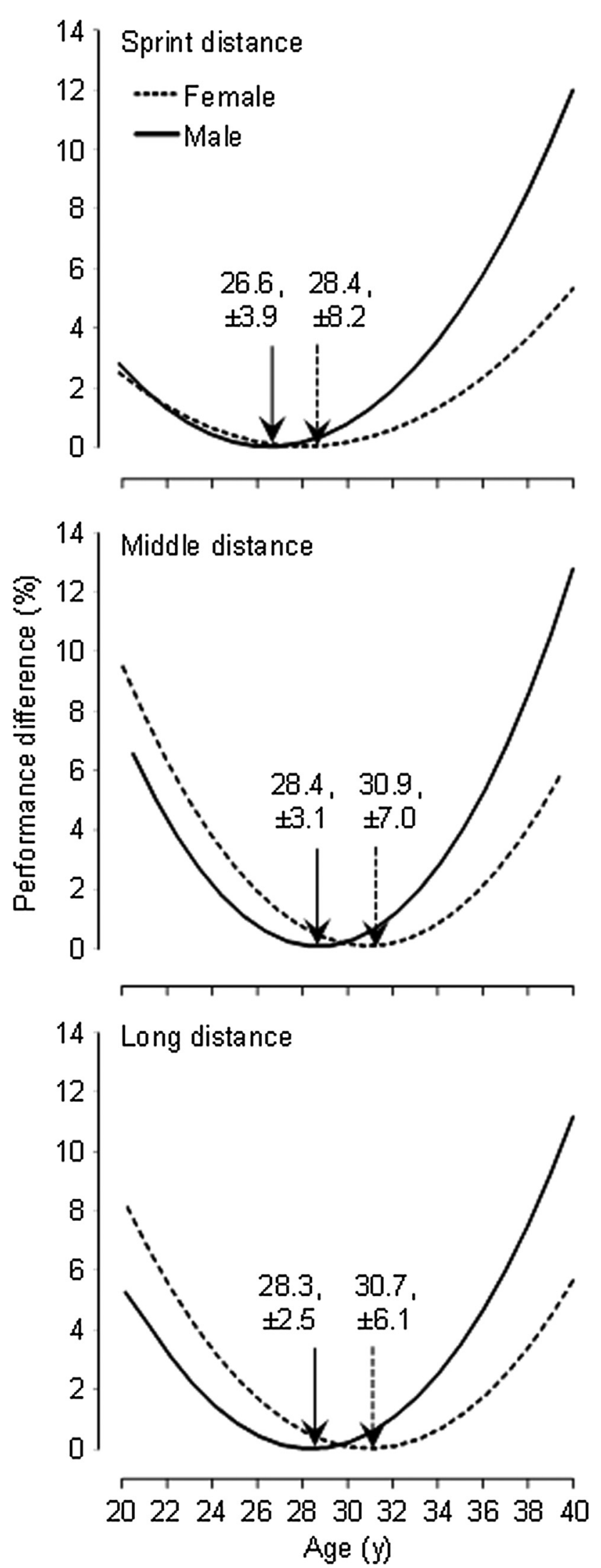

FIGURE 1-The effect of age on performance times modeled as a quadratic and age of peak performance $( \pm 90 \%$ confidence limit) from analyses using mixed linear models on the separate events for the female and male orienteering athletes.
Despite adjusting for race length, distance climbed, number of controls, and home advantage, the magnitude of the differences in mean finishing times between venues was noteworthy, being very large for the sprint events and longdistance male races. Again, these findings indicate some benefit for athletes to train in the area where the WOC is to be held to permit acclimatization and familiarization. The extent of differences between venues also supports our previous statement that factors other than those included in our analyses must influence orienteering performance, such as altitude, temperature, and terrain. On the other hand, differences in finishing times between qualification rounds within a given venue were relatively small, indicating that course planners have been successful thus far at developing qualification rounds of similar difficulty, thereby providing no marked course advantage or disadvantage to athletes racing in different qualification rounds.

Our statistical model estimated the effect of various course characteristics on WOC finishing times, namely that of race length, distance climbed, and number of controls. A $20 \%$ increase in race length had moderate to large slowing effects on performance times during the final rounds of the middle distance events. In contrast, the effect of race length on finishing times in all other final rounds was unclear. The clarity of the race length effect in the final rounds of the middle distance events compared to the others may be associated with the specific physiological factors (4) and pacing strategies (43) attributed to these events. Furthermore, the international competition rules for foot orienteering (18) require that the middle discipline involve consistently technically difficult controls and complex terrain, rendering it the most technically challenging and, consequently, more susceptible to performance differences with length changes. In other words, depending on the terrain difficulty, increasing race distance will affect finishing times differently. It is likely that course setters compensate for easier terrain by lengthening the course, thereby offsetting the effect of distance on shorter races. Future studies might seek to specifically investigate the effect of race length on pacing and finishing times in competitive orienteering to further inform on appropriate training and preparation for courses of differing length.

That adding $50 \mathrm{~m}$ to the distance climbed had the greatest effect on sprint-race performances and the least on long-race performances seems intuitive considering the more pronounced relative increase in climb per race length incurred in sprint races. Compared to running on level ground, running uphill requires a higher energy expenditure (36), more pronounced lower-extremity muscle activation (5), and greater power generation (38), which can explain the decrease in running performance uphill for a given race length. On the other hand, the effect of adding five controls on WOC finishing times was much less clear, potentially quickening, slowing, or not affecting times. Such uncertainty on the effect of controls might be due to their actual placement, which might either facilitate or challenge sighting. Nevertheless, our estimates on how various manipulations to course layouts can affect 
race times provide valuable information to course setters, allowing them to modify certain aspects of a course so that race-day finishing times more closely correspond to the competition rules established by the International Orienteering Federation (18).

The age of peak performance for both male and female orienteers ranged from 27 to 31 yr. Consistent with international track and field athletes $(16,39)$, the age of peak WOC performance was the lowest when involving sprint events (i.e., shortest race length). However, this age was approximately 2-3 yr higher in orienteering compared to runningonly-based sports, which might reflect the relatively longer distance ran in sprint orienteering than track races, importance of cognition in orienteering (19), time required to develop navigational skills and acquire orienteering experience (13), and years needed to reach expert-level sport performance (17). The age of peak performance determined here further reflects that observed in longer-distance races, such as the marathon $(2,27)$.

In exploring the effects of age and sex on the orienteering speed of British competitors, Bird et al. (3) reported no marked decline in performance for males $(0.5 \%-4.2 \%$ per decade) from 21 to $40 \mathrm{yr}$ but reported a moderate decline for females ( $4.7 \%-10 \%$ per decade). In contrast, using our quadratic equation, we estimate that WOC performances decline by $7 \%-9 \%$ per decade in males and $4 \%-7 \%$ per decade in females from their established age of peak performance. The divergent findings between studies are likely due to differences in population, years of competition, and analytical method used. Whereas Bird et al. (3) considered the top 3 male and female performances in 12 different age groups from four British orienteering competitions (two in 1997 and two in 1999), we extracted data from all WOC finishers from 2006 to 2013 who competed in at least two championships. In view of our large sample size of world-class orienteering competitors and advanced statistical modelling approach, we believe our research findings to be further representative of the age of peak performance in orienteering and anticipated performance decline over a $10-y r$ span, at least in relation to World Championships.

The predictability of performance assessed here using ICCs was extremely high within years, suggesting that an athlete who performed well in the qualification round was likely to perform well in the subsequent final event. The predictability of performance from qualification-to-qualification round between years was higher than that from final-to-final due to the between-athlete differences being relatively larger than the within-athlete differences in the qualification rounds. Practically, these results indicate that athletes who finish in the top tier of their qualification rounds one year are likely to finish in the top tier of their qualification rounds another year. However, between years, the ranking of an athlete in the final round is less certain.

Data from only eight WOC were extracted and analyzed herein, which might limit the precision of our estimates. However, data before 2006 were challenging to locate. We also excluded five performance times that were clear outliers, assuming that these data reflected athletes getting lost. Overall, the removal of these outliers has minimal effect on the results and conclusions of our study, although our estimates might not apply to those orienteers who do indeed become lost during WOC races.

Admittedly, our work has direct implications for the foot orienteering community and lesser impact on other sports. Nonetheless, our study results are relevant to the wider orienteering community, such as ski, mountain bike, and trail orienteers, which are all sporting activities represented by the International Orienteering Federation. For instance, estimates of the within-athlete variability in ski orienteering are more likely to resemble ours derived from foot orienteers $(3.2 \%-7.0 \%)$ than those obtained from cross-country skiers $(1.1 \%-1.8 \%)(41)$. Hence, elite ski orienteers should focus on interventions that entail improvements of at least $1 \%$ for smallest worthwhile enhancement in performance. Furthermore, we believe that our rigorous analytical approach provides a sound template for future research in sport science.

\section{CONCLUSIONS}

The present study provides novel insights into the performance of high-level orienteering athletes with respect to the smallest worthwhile enhancement in performance, age of peak performance, and home advantage. Orienteering athletes, coaches, and researchers should focus on and investigate interventions that result in improvements at least equal to the smallest worthwhile enhancements determined here. In addition, the age of peak performance would support focusing on sprint races with younger orienteers and on longer events with more mature athletes, which may be effective in fostering the talent of orienteering athletes. The presence of a clear home advantage suggests that nations hosting championships are likely to benefit from an improved performance and lends support to national teams travelling to competition sites before WOC for familiarization. Increasing the race length, number of controls, and distance climbed had clear effects on performance times in certain WOC events, but not consistently. We recommend that future studies explore the effect of climatic conditions, altitude, and terrain on WOC finishing times and encourage the International Orienteering Federation to standardize documentation of such conditions to facilitate between-venue comparisons and research in this area.

The authors acknowledge the assistance of Peter Öberg and Jan Kocbach, Ph.D., during data collection. We also thank Professor Hans-Christer Holmberg, Director of the Swedish Winter Sports Research Centre (primary site of this work), for facilitating the interactions required for the completion of this work.

The authors have no relevant conflicts of interest to declare. The authors acknowledge the financial support from the Swedish Winter Sports Research Centre, the Swedish Orienteer Federation, and the Swedish Sports Confederation. The results of the present study do not constitute endorsement by the American College of Sports Medicine. 


\section{REFERENCES}

1. Anderson T. Biomechanics and running economy. Sports Med. 1996;22(2):76-89.

2. Berthelot G, Len S, Hellard P, et al. Exponential growth combined with exponential decline explains lifetime performance evolution in individual and human species. Age (Dordr). 2012;34(4):1001-9.

3. Bird SR, Balmer J, Olds T, et al. Differences between the sexes and age-related changes in orienteering speed. J Sports Sci. 2001; 19(4):243-52.

4. Brandon LJ. Physiological factors associated with middle distance running performance. Sports Med. 1995;19(4):268-77.

5. Cai ZY, Hsu CC, Su CP, et al. Comparison of lower limb muscle activation during downhill, level and uphill running. Isokinet Exerc Sci. 2010;18(3):163-8.

6. Carré J, Muir C, Belanger J, et al. Pre-competition hormonal and psychological levels of elite hockey players: relationship to the 'home advantage'. Physiol Behav. 2006;89(3):392-8.

7. Carron AV, Eys MA. Group territory. In: Group Dynamics in Sport. Morgantown (WV): Fitness Information Technology, Incorporated; 2012. p. 418.

8. Carron AV, Loughhead TM, Bray SR. The home advantage in sport competitions: Courneya and Carron's (1992) conceptual framework a decade later. J Sports Sci. 2005;23(4):395-407.

9. Cheuvront SN, Carter R III, DeRuisseau KC, et al. Running performance differences between men and women. Sports Med. 2005; 35(12):1017-24.

10. Coluccia E, Louse G. Gender differences in spatial orientation: a review. J Environ Psychol. 2004;24(3):329-40.

11. Courneya KS, Carron AV. The home advantage in sport competitions: a literature review. J Sport Exerc Psychol. 1992;14:13-27.

12. Eccles DW. Experts' circumvention of processing limitations: an example from the sport of orienteering. Military Psychol. 2008; 20(1 Suppl):S103-21.

13. Eccles DW, Arsal G. How do they make it look so easy? The expert orienteer's cognitive advantage. J Sports Sci. 2015;33(6): 609-15.

14. Eccles DW, Walsh SE, Ingledew DK. A grounded theory of expert cognition in orienteering. J Sport Exerc Psychol. 2002; 24(1):68-88.

15. Eccles DW, Walsh SE, Ingledew DK. Visual attention in orienteers at different levels of experience. J Sports Sci. 2006;24(1):77-87.

16. Ericsson AK. Peak performance and age: an examination of peak performance in sports. In: Baltes PB, Baltes MM, editors. Successful Aging: Perspectives from the Behavioral Sciences. New York (NY): Press Sundicate of the University of Cambridge; 1993. pp. 164-96.

17. Ericsson KA, Lehmann AC. Expert and exceptional performance: evidence of maximal adaptation to task constraints. Annu Rev Psychol. 1996;47:273-305.

18. International Orienteering Federation. Competition Rules for International Orienteering Federation (IOF) Foot Orienteering. 2014. pp. 1-49. Available at: http://www.orienteering.org/. Accessed November 11, 2013.

19. Guzmán Luján JF, Pablos AM, Pablos C. Perceptual-cognitive skills and performance in orienteering. Percept Mot Skills. 2008; 107(1):159-64.

20. Hansen M, Bangsbo J, Jensen J, et al. Effect of whey protein hydrolysate on performance and recovery of top-class orienteering runners. Int J Sport Nutr Exerc Metab. 2015;25(2):97-109.

21. Hébert-Losier K, Jensen K, Holmberg H-C. Jumping and hopping in elite and amateur orienteering athletes and correlations to sprinting and running. Int J Sports Physiol Perf. 2014;9:993-9.
22. Hébert-Losier K, Jensen K, Mourot L, et al. The influence of surface on the running velocities of elite and amateur orienteer athletes. Scand J Med Sci Sports. 2014;24(6):e448-55.

23. Hollings SC, Hopkins WG, Hume PA. Environmental and venuerelated factors affecting the performance of elite male track athletes. Eur J Sport Sci. 2012;12(3):201-6.

24. Hopkins WG, Hawley JA, Burke LM. Design and analysis of research on sport performance enhancement. Med Sci Sports Exerc. 1999;31(3):472-85.

25. Hopkins WG, Hewson DJ. Variability of competitive performance of distance runners. Med Sci Sports Exerc. 2001;33(9):1588-92.

26. Hopkins WG, Marshall SW, Batterham AM, et al. Progressive statistics for studies in sports medicine and exercise science. Med Sci Sports Exerc. 2009;41(1):3-13.

27. Hunter SK, Stevens AA, Magennis K, et al. Is there a sex difference in the age of elite marathon runners? Med Sci Sports Exerc. 2011;43(4):656-64.

28. Jensen K, Johansen L, Kärkkäinen OP. Economy in track runners and orienteers during path and terrain running. J Sports Sci. 1999; 17(12):945-50.

29. Jones MB. The home advantage in individual sports: an augmented review. Psychol Sport Exerc. 2013;14(3):397-404.

30. Knopfli B, Calvert R, Bar-Or O, et al. Competition performance and basal nocturnal catecholamine excretion in cross-country skiers. Med Sci Sports Exerc. 2001;33(7):1228-32.

31. Knowlton RG, Ackerman KJ, Fitzgerald PI, et al. Physiological and performance characteristics of United States championship class orienteers. Med Sci Sports Exerc. 1980;12(3):164-9.

32. Kujala U, Heinonen $\mathrm{O}$, Kvist M, et al. Orienteering performance and ingestion of glucose and glucose polymers. Br J Sports Med. 1989;23(2):105-8.

33. Lebrun CM, McKenzie DC, Prior JC, et al. Effects of menstrual cycle phase on athletic performance. Med Sci Sports Exerc. 1995; 27(3):437-44.

34. Macquet AC, Eccles DW, Barraux E. What makes an orienteer an expert? A case study of a highly elite orienteer's concerns in the course of competition. J Sports Sci. 2012;30(1):91-9.

35. McCutcheon L. The home advantage in high school athletics. J Sport Behav. 1984;7(4):135-8.

36. Minetti AE, Moia C, Roi GS, et al. Energy cost of walking and running at extreme uphill and downhill slopes. J Appl Physiol. 2002; 93(3): 1039-46.

37. Paton CD, Hopkins WG. Variation in performance of elite cyclists from race to race. Eur J Sport Sci. 2006;6(1):25-31.

38. Roberts TJ, Belliveau RA. Sources of mechanical power for uphill running in humans. J Exp Biol. 2005;208(10):1963-70.

39. Schulz R, Curnow C. Peak performance and age among superathletes: track and field, swimming, baseball, tennis, and golf. J Gerontol. 1988;43(5):113-20.

40. Smith TB, Hopkins WG. Variability and predictability of finals times of elite rowers. Med Sci Sports Exerc. 2011;43(11):2155-60.

41. Spencer M, Losnegard T, Hallén J, et al. Variability and predictability of performance times of elite cross-country skiers. Int $J$ Sports Physiol Perf. 2014;9(1):5-11.

42. Sterne JA, Davey Smith G. Sifting the evidence-what's wrong with significance tests? Br Med J. 2001;322(7280):226-31.

43. Tucker R, Lambert MI, Noakes TD. An analysis of pacing strategies during men's world-record performances in track athletics. Int J Sports Physiol Perf. 2006;1(3):233-45.

44. Walsh NP, Whitham M. Exercising in environmental extremes. Sports Med. 2006;36(11):941-76. 